\title{
Search For Dark Matter produced in association With a Higgs Boson in the four lepton final state at $13 \mathrm{TeV}$ With CMS Experiment
}

\author{
Reham Aly* on behalf of the CMS Collaboration \\ Università and INFN Bari \\ E-mail: reham.aly@cern.ch
}

\begin{abstract}
A search for dark matter (DM) particle produced in association with a Higgs Boson $(\mathrm{H})$, where the Higgs boson decays to pair of $\mathrm{Z}$ bosons and each $\mathrm{Z}$ decays to two leptons $(e, \mu)$ is presented. This is the first search for DM particles through this decay channel. This channel has the advantage over other $\mathrm{H}$ decay modes of good mass resolution and a clean reconstruction of the final state particles. The search is performed using data collected by CMS experiment in proton-proton collisions during 2016 with an integrated luminosity of $35.9 \mathrm{fb}^{-1}$ at a center of mass energy of $13 \mathrm{TeV}$. Two simplified benchmark models are used for interpreting the results. No evidence of dark matter candidates is found.
\end{abstract}

European Physical Society Conference on High Energy Physics - EPS-HEP2019 -

10-17 July, 2019

Ghent, Belgium

\footnotetext{
${ }^{*}$ Speaker.
} 


\section{Introduction}

Astrophysical observations indicate that most of the matter in our universe is dark matter (DM) [1]. Till now the nature of DM particles remains unknown and cannot be explained within the standard model (SM). The discovery of the Higgs boson (H) in 2012 by ATLAS [2] and CMS [3] experiments, which represents one of the most important events occurred in the latest decade of Particle Physics history, opens a new door for the DM search at Colliders. The search for DM candidates at colliders is performed through their recoil against a SM particle $X(X=g, q, \gamma, W, Z$, or $H)$, this search known as mono- $X$ signature. If the DM particles are produced at the collider, they would escape from the detector and carry away amount of energy and momentum after the collision. This amount of "missing" energy is used to infer their existence. The Mono-Higgs channel has advantage over than the other mono-X channels that the emission of the Higgs boson as initial state radiation form quark or gluon is highly suppressed which means that the Mono-Higgs channel can probe the new interaction between DM and SM particles.

\section{The Signal Benchmark Models}

Two benchmark simplified models have been considered in the study. The first model is a type II two Higgs doublet model extended by a $Z^{\prime}$ boson " $Z^{\prime} 2 H D M "$ ", shown in Figure 1 (left), where a resonance $Z^{\prime}$ is produced and decays into a Higgs boson and a pseudoscalar $A_{0}$ which in turn decays to a pair of particle-antiparticle DM candidates $(\chi \bar{\chi})$. The second model is the the $Z^{\prime}$ Baryonic, shown in Figure 1(right), where a mediator $Z_{B}^{\prime}$ decays into DM $(\chi \bar{\chi})$ after radiating a Higgs boson [4][5].
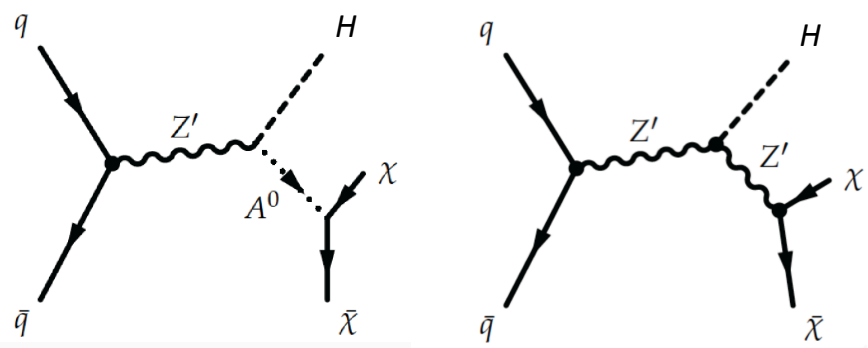

Figure 1: Feyman diagrams for $Z^{\prime} 2 H D M$ (left) and $Z^{\prime}$ Baryonic models (right). Here, $\mathrm{H}$ is the SM Higgs boson, and $\chi$ is the DM candidate.

\section{Analysis Strategy and Event Selection}

The analysis strategy is based on reconstruction of the SM Higgs boson from four charged leptons $(4 e, 4 \mu, 2 e 2 \mu)$ [6], which is considered as a tag for the analysis, in addition a large amount of missing transverse energy to take into account the contribution from the DM candidate production. The analysis flow starts with including pre-selection requirements that must be applied to ensure choosing good object candidates used to reconstruct the Higgs Boson and to ensure that the leptons come from the same primary vertex. Starting from this set of good objects, all possible $\mathrm{Z}$ candidates from opposite charge and same flavor leptons are built with mass between 12 and 120 
$\mathrm{GeV}$, including also the contribution of final state radiation from any lepton if exists. Among all those built $\mathrm{Z}$ candidates, all possible $\mathrm{ZZ}$ candidates with non overlapping leptons are built. The $\mathrm{Z}$ candidate with reconstructed mass closest to the nominal $\mathrm{Z}$ boson mass is denoted as $Z_{1}$, and the second one is denoted as $Z_{2}$. To remove any contribution from QCD production of low mass resonance, all possible opposite-sign pair of leptons "regardless of lepton flavor" that can be built must satisfy $M_{l l}>4 \mathrm{GeV}$. All the leptons used to select the $Z Z$ candidates must be separated by $\Delta R\left(l_{i}, l_{j}\right)>0.02$. The mass of the four reconstructed leptons should be greater than $70 \mathrm{GeV}$ and the mass of the first $Z$ candidate " $Z_{1}$ " greater than $40 \mathrm{GeV}$. If more than one $\mathrm{ZZ}$ candidate survives, the one with the highest value of the scalar sum of the 4-lepton $\mathrm{pT}$ is chosen. The reconstructed 4 leptons invariant mass is shown in Figure 2 left as obtained from data, simulated background and signal samples. A peak around $125 \mathrm{GeV}$ is clearly observed in data and in the signal as expected, this peak is compatible with the prediction of the SM Higgs boson and confirms the discovery made in 2012. But due to the low signal cross section, the signal is completely overwhelmed by background at this step so an additional selections are required to suppress the background level. For this purpose we require that the invariant mass of the four leptons is around the Higgs peak within $10 \mathrm{GeV}$, the number of the four leptons to be exactly four and the number of b-tagged jets to be less than two. Those requirements reduce the contribution of $t \bar{t}+V(\mathrm{~V}=\mathrm{Z}$ or $\mathrm{W}), V V V, Z H$ and $W H$ background events without a relevant impact on the signal. The final missing transverse energy $\left(p_{T}^{m i s s}\right)$ distribution after applying all the selection cuts is shown in Figure 2 right as obtained from data, simulated background samples, described in the next section, and DM signal with two different mass points. The ratio of the data and the sum of all the backgrounds is shown in the bottom. The hatched band correspond to the systematic uncertainty.
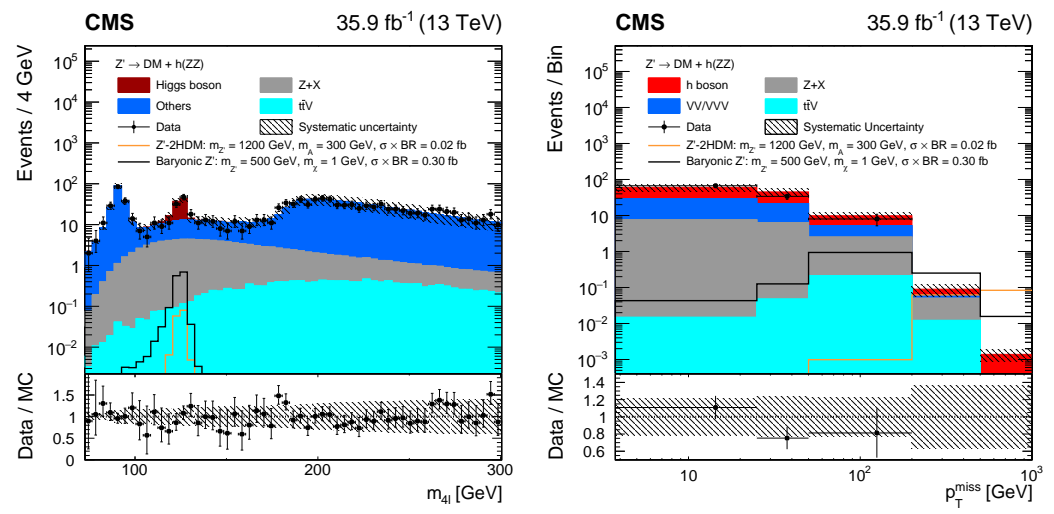

Figure 2: The four leptons invariant mass distribution (left) obtained after the SM Higgs selection and the final $p_{T}^{\text {miss }}$ distribution (right) obtained after all the selections for data "solid points", MC background processes (colored stacked histograms) and two benchmark signals Z'-2HDM (orange line) and baryonic $Z^{\prime}$ (black line) model.

\section{Background}

The dominant irreducible backgrounds come from the SM Higgs boson and the non-resonant $\mathrm{ZZ}$ production which is determined from simulation. Beside this irreducible background another contribution comes from background dominated by $Z+$ jets called " $Z+X$ " background which is 
determined from data. This background arises from secondary leptons misidentified as prompt leptons from heavy flavor hadron or light mesons decay within jets. The non prompt lepton background also contains smaller contributions from $t \bar{t}+$ jets, $Z \gamma+j e t s, W Z+j e t s$, and $W W+j e t s$ events, with a jet misidentified as a prompt lepton. The $\mathrm{Z}+\mathrm{X}$ background is estimated from data by determining the lepton misidentification probability in a dedicated control region "CR" and then using it to derive the background contribution in the SR. The lepton misidentification probability is defined as the probability that a lepton passing a loose selection criteria to also passes the tight selection criteria. The misidentification probability is measured in a Z+lepton CR as functions of the lepton candidate $\mathrm{pT}$, its location in the detector "barrel or endcap region" and $p_{T}^{\text {miss }}$ in the event. Applying the lepton misidentification probabilities relies on defining two CRs. One region is expected to be populated with events that have only two prompt leptons (mostly from DY production, with a small contribution from $t \bar{t}$ and $Z \gamma$ events) and the second region is expected to be populated with WZ+jets events with three prompt leptons plus the type of events that populate the first CR but with different properties.

\section{Results}

After the final analysis selection, no significant excess of data are observed over the SM predictions. The observed and expected upper limits on the DM candidate production cross section are set as shown in Figure 3 for the $Z^{\prime}-2 H D M$ with $m_{A}=300 \mathrm{GeV}$ (left) and for the $Z^{\prime}$ baryonic model with $m_{\chi}=1 \mathrm{GeV}$ (right). The upper limits already include the statistical combination of the three final states. No mass points of the parameter space of the two models can be excluded at $95 \%$ $\mathrm{CL}$. The results from $Z Z \rightarrow 4 l$ decay channel combined with other Higgs decay channels $(b \bar{b}, \gamma \gamma$, $\tau \tau$ and $W W$ ). The observed and expected $95 \%$ CL exclusion contours are set on the signal strength $\left(\sigma / \sigma_{t h}\right)$ in the $m_{Z^{\prime}}-m_{A}$ and $m_{Z^{\prime}}-m_{\chi}$ planes for the $Z^{\prime}-2 H D M$ Figure 4 (left) and baryonic $Z^{\prime}$ Figure 4 (right) model respectively [7]. The combined analysis can exclude at $95 \% \mathrm{CL}$ the range of $m_{Z^{\prime}}$ from 500 to $3200 \mathrm{GeV}$ for $m_{A}=300 \mathrm{GeV}$ and the range of $m_{Z^{\prime}}$ from 100 to $1600 \mathrm{GeV}$ for $m_{\chi}=1 \mathrm{GeV}$.
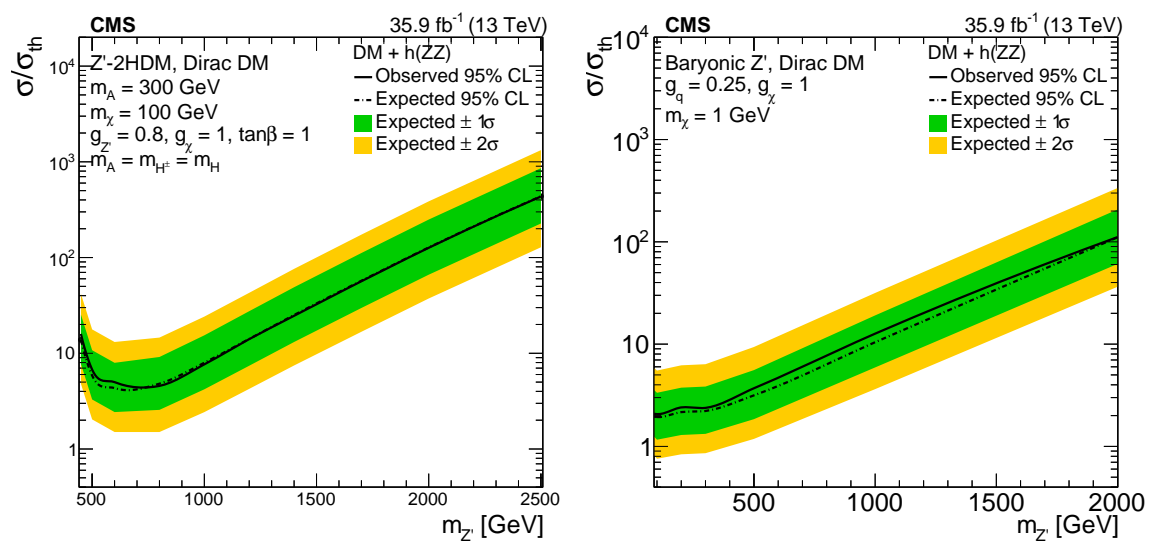

Figure 3: The upper limits on the observed and expected DM production cross section at 95\% CL for the Z'-2HDM with $m_{A}=300 \mathrm{GeV}$ (left) and for the baryonic Z' with $m_{\chi}=1 \mathrm{GeV}$ (right) model. The inner and outer shaded bands show the 68 and $95 \%$ uncertainties in the expected limit, respectively. 

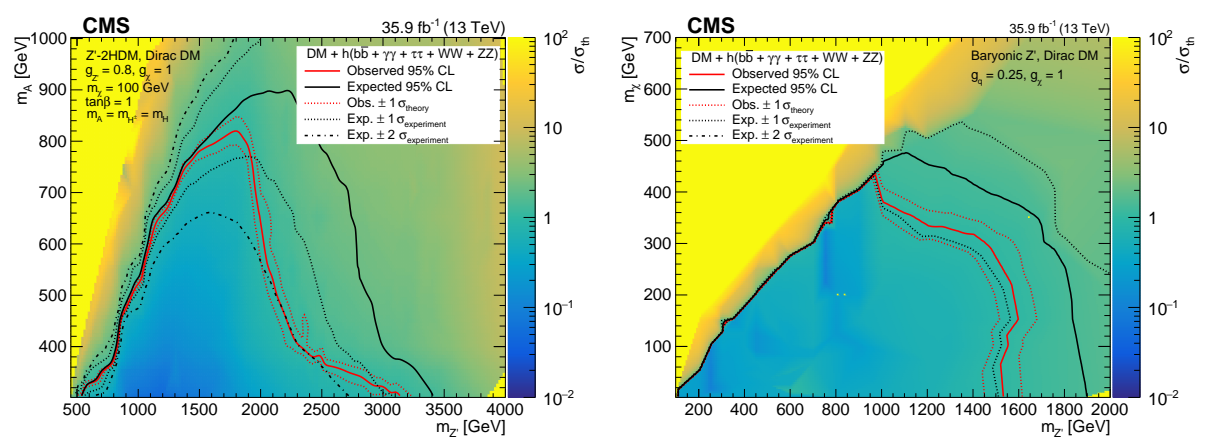

Figure 4: The upper limits at $95 \% \mathrm{CL}$ on the observed and expected $\sigma / \sigma_{t h}$ in the $m_{Z^{\prime}}-m_{A}$ and $m_{Z^{\prime}}-m_{\chi}$ planes for the Z'-2HDM (left) and baryonic Z' model (right) respectively.

\section{Summary}

A search for dark matter particles produced in association with a Higgs boson decaying to four leptons has been presented using data collected from CMS experiment at a center-of-mass energy of $13 \mathrm{TeV}$ and corresponding to an integrated luminosity of $35.9 \mathrm{fb}^{-1}$. Results from Higgs ZZ decay channel is presented as well as the results from the combination with other decay channels of the Higgs boson ( $b \bar{b}, \gamma \gamma, \tau \bar{\tau}$ and $W^{+} W^{-}$). No significant deviation from the standard model prediction is observed in the Higgs $\mathrm{ZZ}$ channel or in the combined channels. Upper limits at $95 \%$ confidence level on the production cross section of dark matter are set in context of a Z'2HDM and in a baryonic Z' model.

\section{References}

[1] V. Trimble, Existence and nature of dark matter in the universe, Ann. Rev. Astron. Astrophys. 25 (1987) 425, doi:10.1146/annurev.aa.25.090187.002233.

[2] ATLAS Collaboration, Observation of a new particle in the search for the standard model Higgs boson with the ATLAS detector at the LHC, Phys. Lett. B 716 (2012) 1, doi:10.1016/j.physletb.2012.08.020, arXiv:1207.7214.

[3] CMS Collaboration, Observation of a new boson with mass near $125 \mathrm{GeV}$ in pp collisions at $\sqrt{(s)}=$ 7 and 8 TeV, JHEP 06 (2013) 081,doi:10.1007/JHEP06(2013)081, arXiv:1303.4571.

[4] L. Carpenter et al, Mono-Higgs: a new collider probe of dark matter, Phys.Rev. D 89 (2014) 075017, doi:10.1103/PhysRevD.89.075017, arXiv:1312.2592

[5] Abercrombie et al., Dark Matter Benchmark Models for Early LHC Run-2 Searches: Report of the ATLAS/CMS Dark Matter Forum, arXiv:1507.00966v1 [hep-ex], 2015

[6] CMS Collaboration, Measurements of properties of the Higgs boson decaying into the four-lepton final state in pp collisions at $\sqrt{(s)}=13 \mathrm{TeV}$, doi:10.1007/JHEP11(2017)047, arXiv:1706.09936 [hep-ex]

[7] CMS Collaboration, Search for dark matter particles produced in association with a Higgs boson in proton-proton collisions at $\sqrt{(s)}=13 \mathrm{TeV}$, CMS PAPER EXO-18-011, 2019. 